\title{
Retinotoxicity of Hydroxychloroquine: Is It Possible to Demonstrate by Spectral Domain Optical Coherence Tomography Before Development? A Cross Sectional Investigation
}

\author{
Hasan Fatih ÇAY, ${ }^{1}$ Muhammet Kazım EROL, ${ }^{2}$ Deniz TURGUT ÇOBAN, ${ }^{2}$ Mehmet BULUT, ${ }^{2}$ \\ İlhan SEZER, ${ }^{1}$ Tuncay ÇAKIR, ${ }^{3}$ Naciye Füsun TORAMAN ${ }^{3}$ \\ ${ }^{2}$ Department of Physical Medicine and Rehabilitation, Antalya Training and Research Hospital, Division of Rheumatology, Antalya, Turkey \\ ${ }^{2}$ Department of Ophthalmology, Antalya Training and Research Hospital, Antalya, Turkey \\ ${ }^{3}$ Department of Physical Medicine and Rehabilitation, Antalya Training and Research Hospital, Antalya, Turkey
}

\begin{abstract}
Objectives: This study aims to evaluate the alterations of retinal layers in rheumatic patients treated with hydroxychloroquine but without the signs or symptoms of retinopathy by using spectral domain ocular coherence tomography (SD-OCT).

Patients and methods: The retinal layers of a total of 402 eyes including 114 patients treated with hydroxychloroquine (for rheumatoid arthritis $(n=40)$, Sjögren's syndrome $(n=47)$ and connective tissue diseases $(n=27)$ and age-matched 87 healthy controls were evaluated with SD-OCT. The macular cube protocol, optic disc cube protocol and horizontal and vertical HD 5-line raster scan protocol were applied. The measured parameters were compared between hydroxychloroquine users and healthy control group. The results of these parameters were also compared with other disease groups using hydroxychloroquine. The correlation of these parameters with the duration of drug consumption and dose was assessed.

Results: All layers of outer fovea, superior and inferior quadrants of retinal nerve fiber layers of hydroxychloroquine users were thinner than nonusers. Connective tissue disease group had longer duration and higher cumulative dose of hydroxychloroquine than other diagnostic groups. This group had thinner mean retinal nerve fiber layers values than the other groups as well. There were significant and negative correlations between cumulative dose of drug and parafoveal region thickness of outer fovea and inferior quadrant of retinal nerve fiber layers. Thickness of parafoveal and perifoveal layers was negatively correlated with the dose of drug per kg of body weight.

Conclusion: Our study results show that SD-OCT may be the golden standard technique for the follow-up of antimalarial-induced retinotoxicity in future.

Key words: Hydroxychloroquine; retinotoxicity; spectral domain ocular coherence tomography.
\end{abstract}

Chloroquine (CQ) and hydroxychloroquine (HCQ) are anti-malarial drugs which have been used since 1950 to treat auto-inflammatory diseases such as rheumatoid arthritis (RA), and connective tissue diseases (CTDs) including systemic lupus erythematosus, Sjögren's syndrome $(\mathrm{SjS})$ and dermatomyositis. ${ }^{1}$ The ocular toxicity associated with these drugs was first described in 1957.2 Although HCQassociated retinotoxicity is a rare phenomenon, this drug is not free of the potentially irreversible adverse effect.1,3,4 Anti-malarial-induced ocular toxicity is characterized by bilateral pigmentary alteration of the macula, often sparing the foveal centre (bull's eye maculopathy). It may be seen infrequently in the peripheral retina without significant macular alterations, central visual loss, visual field defects and color vision defects. Early detection of the developing retinotoxicity is essential to prevent irreversible consequences. ${ }^{4}$ The American Academy of Ophtalmology has defined a roadmap for the management of 
anti-malarial-induced retinotoxicity based on predefined risk factors. ${ }^{5}$ Visual field analysis, electroretinography, fundus autoflorescence, and fundus fluorescent angiography are the methods used to evaluate retinal alterations.

Optical coherence tomography (OCT) is the primary technique for the evaluation of retinal diseases. It has been recently evolved into the spectral-domain OCT (SD-OCT), which has a 43-100 times faster imaging capacity and higher signal-to noise ratio compared to the time-domain OCT. The inner segment and outer segment lines, external limiting membrane and the inner retinal structure including retinal nerve fiber layer (RNFL) and plexiform layer can be visualized more clearly by SD-OCT with $5 \mu \mathrm{m}$ axial resolution. Spectral-domain OCT also allows for automatic and manual measurement of the retinal layers and RNFL. ${ }^{6-8}$

In this study, we aimed to measure the retinal layer thicknesses in patients using HCQ and to investigate whether retinal alterations were able to be detected before symptoms and/or signs of retinotoxicity became evident by using SD-OCT.

\section{PATIENTS AND METHODS}

A total of 114 female patients on HCQ for the treatment of RA $(n=40), \operatorname{SjS}(n=47)$ and CTDs $(n=27)$, including systemic lupus erythematosus and other undefined connective tissue disorders were enrolled in this study. They were selected according to the following inclusion criteria: being 18 years old or older, having less than \pm 6 diopter sphere of refractive error and having the ability to perform visual field testing. Exclusion criteria were as follows: history of $\mathrm{CQ}$ consumption, presence of diabetes mellitus, thyroid dysfunction, cataracts, glaucoma, and a history of uveitis or vascular disease which might compromise the ocular structures. Additionally, patients who had any macular autofluorescent abnormality demonstrated by 45 degree fundus autoflorescence imaging and/or any central or paracentral scotoma in central visual field testing were excluded. The SD-OCT database of the Ophthalmology Department of our Institution was used to identify a healthy control group of 87 age-matched females. The mean age of the healthy controls was 46.7 (95\% CI: 45.6-47.9) years. On the other hand, the mean ages of HCQ-users were 46.7 (95\% CI: 43.8-49.6); 48.7 (95\% CI: 46.5-50.8) and 44.9 (95\% CI: 41.348.5) years for the RA, SjS and CTD groups, respectively.

Demographic data including age, preliminary diagnosis, dose and total duration of $\mathrm{HCQ}$, weight and height of all patients were recorded. The cumulative dose of consumed HCQ and the dose per kilogram of actual body weight and of lean body mass ${ }^{1}$ were calculated. All patients were informed about the study protocol, and their informed consents were obtained. The study was approved by the local ethics committee of the Antalya Training and Research Hospital. A comprehensive ophthalmologic examination was applied for all patients. This examination protocol included the best corrected visual acuity using snellen chart, measurement of intraocular pressure by Goldmann applanation tonometry, 12-degree visual field perimetry with a white stimulus by using Octopus 900 (Interzeag AG, Schlieren- Zurich, Swiss). Fundus autofluorescence imaging and color fundus photography were recorded by using the Visucam NM FA (Carl Zeiss Meditec AG., Jena, Germany) with 45 degree fundus camera mode. The limits for the wavelength of the excitation light were between $510 \mathrm{~nm}$ and $580 \mathrm{~nm}$; and those for the wavelength of the barrier filter were between $650 \mathrm{~nm}$ and $735 \mathrm{~nm}$. At least five autofluorescence imaging shots were taken with a pupil width of at least $6 \mathrm{~mm}$ to obtain high-quality images.

Spectral-domain OCT imaging was applied by using the Cirrus HD OCT (Cirrus Carl Zeiss Meditec Inc., Dublin CA, USA). SD-OCT examination was performed according to three different protocols: the first one was the "macular cube $512 \times 128$ protocol" in which retinal thickness was measured in each of the nine regions quantitatively (Figure 1). The second one was the "optic disc cube protocol" in which the peripapillary retinal nerve fiber thickness was measured in each of four (superior, inferior, nasal, temporal) quadrants. The third protocol was the "horizontal and vertical HD five line raster scan" which comprised of 4,096 axial scan in each line. The distance between each individual scan was $0.5 \mathrm{~mm}$. In this protocol, manual measurement of the outer 

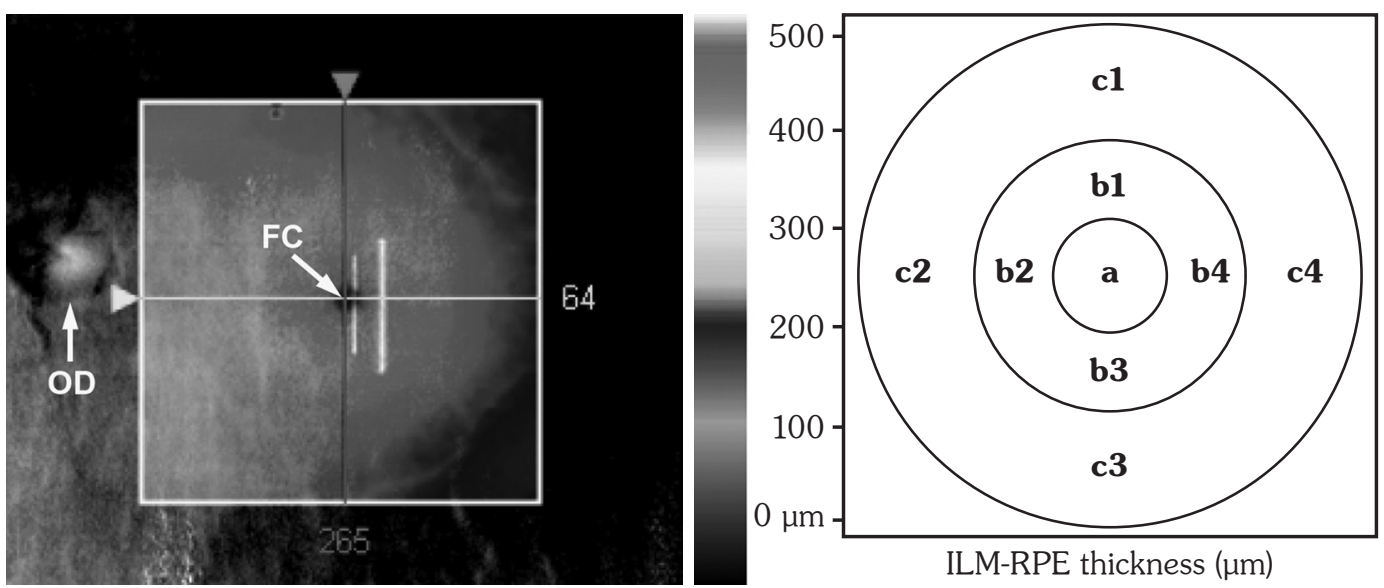

Figure 1. Macular cube 512x128 protocol measurement. OD: Optic disc; FC: Fovea centralis; a: Central fovea, (First circle with diameter of $1 \mathrm{~mm}$ ); b1+b2+b3+b4/4: Full thickness fovea first quadrants, (Second circle with diameter of $3 \mathrm{~mm}$ ); $\mathrm{c} 1+\mathrm{c} 2+\mathrm{c} 3+\mathrm{c} 4 / 4$ : Full thickness fovea second quadrants, (Third circle with diameter of $6 \mathrm{~mm}$ ).

retinal layer thickness (OLT) was performed at a point in the central fovea from internal limiting membrane (ILM) to the apex of retinal pigmented epithelium (RPE) by an ophtalmologist who was blinded to the primary diagnosis and drug consumption properties of the subjects. Additional measurements were performed at points 1 and $2 \mathrm{~mm}$ distance to the central fovea (from the outer plexiform layer to the apex of the retina with pigmented epithelium) in each quadrant by using computer-based caliper measurement tool of the SD-OCT system. The measured thicknesses at 1 and $2 \mathrm{~mm}$ distances from central fovea were named parafovea and perifovea, respectively (Figure 2). The arithmetic means of these two measurements were calculated.

\section{Statistical analysis}

Statistical analysis was performed using SPSS (SPSS Inc., Chicago, IL, USA) for Windows v13.0 software. The Student's t-test was used to compare the means of continuous variables between the two different groups. One-way analysis of variance (ANOVA) with post-hoc Bonferroni correction was used to compare the means of measured parameters among three or more different groups. The Pearson's correlation analysis was used to define the relationship of retinal thicknesses with drug dose and duration of drug consumption. A cut-off value of 0.05 was considered statistically significant.

\section{RESULTS}

There was no significant difference between the mean ages of the study and healthy control groups. The cumulative dose of consumed drug and total duration of drug consumption were higher among the subjects with CTD than in the others (Table 1).

Statistical analysis was performed by considering the measurements of both eyes of

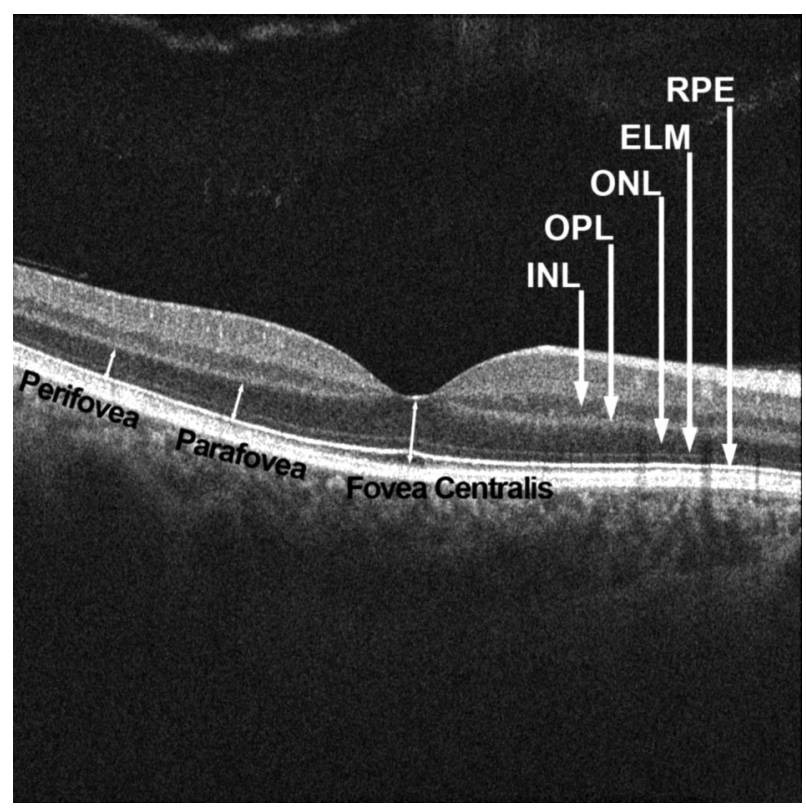

Figure 2. Manual measurement of the outer retinal layer thickness. RPE: Retinal pigmented epithelium; ELM: External limiting membrane; ONL: Outer nuclear layer; OPL: Outer plexiform layer; INL: Inner nuclear layer; Distance between fovea centralis and parafovea measurement site was $1 \mathrm{~mm}$; between fovea centralis and perifoveal measurment site was $2 \mathrm{~mm}$. 
Table 1. Mean ages and drug consuming properties of subjects according to the disease groups

\begin{tabular}{|c|c|c|c|c|c|c|c|c|c|c|}
\hline & \multicolumn{2}{|c|}{$\begin{array}{l}\text { Age } \\
\text { (years) }\end{array}$} & \multicolumn{2}{|c|}{$\begin{array}{l}\text { Treatment duration } \\
\text { (months) }\end{array}$} & \multicolumn{2}{|c|}{$\begin{array}{l}\text { Cumulative dose } \\
\text { (g) }\end{array}$} & \multicolumn{2}{|c|}{$\begin{array}{l}\text { Dose/body } \\
\text { weight }(\mathrm{mg} / \mathrm{kg})\end{array}$} & \multicolumn{2}{|c|}{$\begin{array}{c}\text { Dose/lean body } \\
\text { mass }(\mathrm{mg} / \mathrm{kg})\end{array}$} \\
\hline & Mean \pm SD & $95 \% \mathrm{CI}$ & Mean \pm SD & $95 \% \mathrm{CI}$ & Mean \pm SD & $95 \% \mathrm{CI}$ & Mean $\pm S D$ & $95 \% \mathrm{CI}$ & Mean \pm SD & $95 \% \mathrm{CI}$ \\
\hline $\begin{array}{l}\text { Rheumatoid } \\
\text { arthritis }(n=40)\end{array}$ & $46.7 \pm 13.2$ & $43.8-49.6$ & $13.8 \pm 13.2$ & $10.8-16.8$ & $114.8 \pm 103.9$ & 91.1-138.6 & $3.6 \pm 1.5$ & $3.3-3.9$ & $5,5 \pm 2.1$ & $5.1-6.0$ \\
\hline $\begin{array}{l}\text { Sjögren's } \\
\text { syndrome }(n=47)\end{array}$ & $48.7 \pm 10.6$ & $46.5-50.8$ & $14.1 \pm 14.4$ & $11.0-17.2$ & $103.1 \pm 89.0$ & $84.2-121.9$ & $3.9 \pm 1.8$ & $3.6-4.4$ & $5.4 \pm 1.9$ & $4.9-5.8$ \\
\hline $\begin{array}{l}\text { Connective tissue } \\
\text { disease }(\mathrm{n}=27) \\
\text { Healthy }\end{array}$ & $44.9 \pm 13.2$ & 41.3-48.5 & $24.4 \pm 29.0$ & $15.9-32.8^{*}$ & $180.3 \pm 186.4$ & $126.1-234.4^{* *}$ & $3.6 \pm 1.3$ & $3.2-3.9$ & $5.0 \pm 1.7$ & $4.5-5.5$ \\
\hline controls ( $\mathrm{n}=87$ ) & $46.7 \pm 7.7$ & $45.6-47.9$ & \multicolumn{2}{|c|}{ NA } & \multicolumn{2}{|c|}{ NA } & \multicolumn{2}{|c|}{ NA } & \multicolumn{2}{|c|}{ NA } \\
\hline
\end{tabular}

each subjects. Therefore, the number was doubled for each subjects enrolled in the study. Comparison of the foveal thickness among healthy controls and HCQ-users revealed that the outer foveal segments, in particular, tended to be significantly higher in the healthy control group. The central part of the outer fovea was $169.8 \pm 14.8 \mu \mathrm{m}$ for healthy controls, while that of HCQ-users was $166.0 \pm 14.6 \mu \mathrm{m}(\mathrm{p}=0.013)$. Measurement of the parafovea revealed $117.5 \pm 10.1$ vs. $110.5 \pm 11.0$ $\mu \mathrm{m}$ for the former and latter groups, respectively $(p=0.000001)$ (Table 2). Measurement of the foveal layers revealed that the central area of full foveal thickness was not different among the disease groups. In contrast, the foveal layers were thinner in subjects with CTD compared to healthy controls (Table 3). There was no inner segment/ outer segment band discontinuation in any of the subjects.
Retinal nerve fiber layers of the superior and inferior quadrants were thicker in healthy control subjects compared to HCQ-users (Table 2). When comparison was made among disease groups, RNFL thickness in the superior and inferior quadrants of CTD subjects were $114.7 \mu \mathrm{m}$ (95\% CI: 110.2-119.3) and $117.0 \mu \mathrm{m}$ (95\% CI: 112.0-122.0), respectively. These figures were statistically significantly different from the other groups (Table 4).

The cumulative dose of HCQ was found to be correlated with the parafoveal region thickness of OLT $(r=-0.192, p<0.001)$ and inferior quadrant of RNFL $(\mathrm{r}=-0.104, \mathrm{p}<0.05)$. The OLT in the parafoveal region was found to be correlated with drug dose per $\mathrm{kg}$ of actual body weight and per $\mathrm{kg}$ of lean body mass $(\mathrm{r}=-0.159$ and -0.173 respectively, $\mathrm{p}<0.001)$. Similarly, the OLT in the perifoveal region was found to be correlated with the same parameters (Table 5).

Table 2. Foveal layer thicknesses and retinal nerve fiber layer measurements among healthy controls and hydroxychloroquine users

\begin{tabular}{|c|c|c|c|}
\hline & Healthy controls $(n=174)$ & Hydroxychloroquine users $(n=228)$ & \\
\hline & Mean \pm SD & Mean \pm SD & $p^{*}$ \\
\hline \multicolumn{4}{|l|}{ Full thickness fovea $(\mu \mathrm{m})$} \\
\hline Central thickness & $249.9 \pm 20.0$ & $249.5 \pm 22.5$ & 0.839 \\
\hline First quadrants & $322.3 \pm 14.2$ & $319.5 \pm 16.7$ & 0.084 \\
\hline Second quadrants & $281.3 \pm 11.0$ & $279.45 \pm 16.9$ & 0.209 \\
\hline \multicolumn{4}{|l|}{ Outer fovea $(\mu \mathrm{m})$} \\
\hline Central thickness & $169.8 \pm 14.8$ & $166.0 \pm 14.6$ & 0.013 \\
\hline Parafovea & $117.5 \pm 10.1$ & $110.5 \pm 11.0$ & $<0.001$ \\
\hline Perifovea & $103.8 \pm 7.6$ & $100.0 \pm 8.5$ & $<0.001$ \\
\hline \multicolumn{4}{|c|}{ Retinal nerve fiber layer ( $\mu \mathrm{m})$} \\
\hline Superior quadrant & $122.3 \pm 13.8$ & $118.1 \pm 15.9$ & 0.007 \\
\hline Inferior quadrant & $127.1 \pm 15.2$ & $121.3 \pm 18.4$ & 0.001 \\
\hline Nasal quadrant & $73.1 \pm 11.9$ & $74.3 \pm 14.8$ & 0.384 \\
\hline Temporal quadrant & $64.0 \pm 9.5$ & $65.2 \pm 10.6$ & 0.243 \\
\hline
\end{tabular}




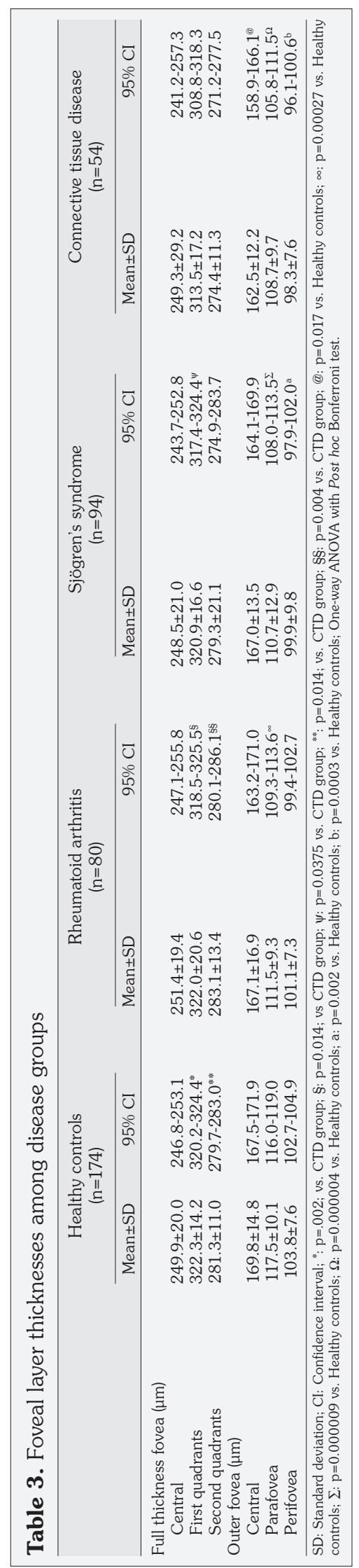

\section{DISCUSSION}

Although the exact pathogenesis of HCQ-induced retinopathy has not been well-established yet, the similarity of the chemical structure and induced-retinopathy of HCQ to CQ suggest that the mechanisms may be analogous. ${ }^{9} \mathrm{CQ}$ binds to melanin of retinal pigmented epithelium with a high concentration and remains there for a prolonged period of time, even after cessation of therapy. ${ }^{10}$ A study demonstrated that $\mathrm{CQ}$ disrupted the lysosomal function in retinal neurons and RPE. ${ }^{11}$ The retinopathy caused by anti-malarial drugs consists of bilateral macular pigmentary alterations and functional abnormalities in the visual field. Ophthalmoscopic examination may reveal granular pigmentary changes in the form of bull's-eye maculopathy with a circle of RPE atrophy surrounding the central fovea. ${ }^{12}$ It was demonstrated that high-speed ultra-high resolution OCT was able to detect the loss of the perifoveal photoreceptor inner segment/ outer segment junction and thinning of the outer nuclear layer in patients receiving HCQ. ${ }^{13}$ Retinal thinning was considered to be the earliest indicator of retinopathy. ${ }^{14}$ Two histopathological studies performed in humans with advanced CQ-induced retinopathy revealed destruction of the cone and rods, sparing the foveal cones. ${ }^{15,16}$ Kahn et al. ${ }^{17}$ compared retinal thicknesses at points 0.5 and $1 \mathrm{~mm}$ from the foveal center in patients with electroretinogram alterations without fundoscopic signs of retinotoxicity, and in age-matched controls. They found significant differences in measurements performed at $1 \mathrm{~mm}$ distance but not at $0.5 \mathrm{~mm}$ distance from the foveal center between the groups. In previous studies, measurements of the retinal structures were applied by accepting all layers as a whole. ${ }^{5,17}$ In the present study, we measured the outer nuclear layer (photoreceptor nucleus layer), inner and outer segments. The layers did not contain any ganglion or nerve fiber. No difference was found in full thickness evaluation. The measurements performed by isolation indicated significant differences between drug users and controls. This might be interpreted as a possible decline in the thickness of these anatomical layers, before the fundoscopic alterations of retinotoxicity became apparent. 
Table 4. Retinal nerve fiber layer thickness of subjects in different disease groups

\begin{tabular}{|c|c|c|c|c|c|c|c|c|}
\hline & \multicolumn{2}{|c|}{$\begin{array}{l}\text { Healthy controls } \\
\qquad(\mathrm{n}=174)\end{array}$} & \multicolumn{2}{|c|}{$\begin{array}{l}\text { Rheumatoid arthritis } \\
\qquad(\mathrm{n}=80)\end{array}$} & \multicolumn{2}{|c|}{$\begin{array}{l}\text { Sjögren's syndrome } \\
\quad(\mathrm{n}=94)\end{array}$} & \multicolumn{2}{|c|}{$\begin{array}{l}\text { Connective tissue diseases } \\
\qquad(\mathrm{n}=54)\end{array}$} \\
\hline & Mean \pm SD & $95 \% \mathrm{CI}$ & Mean \pm SD & $95 \% \mathrm{CI}$ & Mean \pm SD & $95 \% \mathrm{CI}$ & Mean \pm SD & $95 \% \mathrm{CI}$ \\
\hline \multicolumn{9}{|l|}{ RNFL $(\mu \mathrm{m})$} \\
\hline Superior quadrant & $122.3 \pm 13.8$ & $120.2-124.4$ & $117.4 \pm 16.7$ & $113.6-121.3$ & $120.7 \pm 14.5$ & $117.7-123.7$ & $114.7 \pm 16.6$ & $110.2-119.3^{*}$ \\
\hline Inferior quadrant & $127.1 \pm 15.2$ & $124.8-129.5$ & $123.8 \pm 18.7$ & $119.5-128.1$ & $121.8 \pm 18.0$ & $118.0-125.5$ & $117.0 \pm 18.1$ & $112-0-122.0^{* *}$ \\
\hline Nasal quadrant & $73.1 \pm 11.9$ & $71.3-75.0$ & $73.9 \pm 13.0$ & 70.9-76.9 & $75.7 \pm 14.6$ & 72.7-78.7 & $72.4 \pm 17.4$ & $67.6-77.2$ \\
\hline Temporal quadrant & $64.0 \pm 9.5$ & $62.5-65.4$ & $64.8 \pm 10.0$ & $62.6-67.1$ & $65.7 \pm 10.1$ & $63.6-67.8$ & $64.9 \pm 12.4$ & $61.4-68.3$ \\
\hline
\end{tabular}

Despite the finding of no difference in full foveal thicknesses between drug users and nonusers, a negative and significant correlation was present between the thickness of these structures and the dose of drug per kilogram of body weight. However, the clinical implication of this finding remains unknown.

Histological investigations reported that receptors located in the parafoveal region were affected by drug consumption earlier and more intense than in the other parts of the retina. ${ }^{10,16,18}$ Our findings were consistent with these implications. It is known that the parafoveal and perifoveal layers are preferentially involved by anti-malarial-induced retinotoxicity than the foveal layer. The factors, considered as being responsible for this distribution are unknown. In a study performed on retinal structures of nonprimate rats, the external limiting membrane was found to have a barrier-function against diffusion of proteins. ${ }^{19}$ Additionally, loss or weakness of the junctions performing this barrial function was found in fovea centralis. ${ }^{20}$ Therefore, it is reasonable to speculate that there may be diffusion of HCQ in this barrier-free area. However, this claim needs to be clarified by further investigations.

In a previous study which was performed through measurement of the peripapillary RNFL by scanning laser polarimetry, CQ-users were found to have thinner layers compared to healthy controls. ${ }^{21}$ However, it was emphasized that using fixed corneal compensator during this measurement could not eliminate the birefringence, which might impair the acuity of the RNFL measurement. In addition, Pasadhika et al. ${ }^{4}$ reported that the difference in thickness of RNFL was found only in the nasal quadrant of patients treated with CQ compared to healthy controls. In contrast, the thinning of RNFL was localized in the superior and inferior quadrants of HCQ-users in our study. This difference may be a

Table 5. Correlations between ocular coherence tomography measurements and drug consumption parameters

\begin{tabular}{|c|c|c|c|c|}
\hline & Dose/body weight & Dose/lean body mass & Cumulative dose & Treatment duration \\
\hline & \multicolumn{4}{|c|}{ Correlation coefficients $(\mathrm{r})$} \\
\hline \multicolumn{5}{|l|}{ Full thickness fovea } \\
\hline Central & -0.108 & -0.091 & 0.10 & 0.060 \\
\hline First quadrants & $-0.119^{*}$ & -0.071 & -0.081 & -0.002 \\
\hline Second quadrants & $-0.120^{*}$ & -0.053 & -0.039 & 0.016 \\
\hline \multicolumn{5}{|l|}{ Outer fovea } \\
\hline Central & $-0.140^{*}$ & $-0.133^{*}$ & -0.035 & -0.039 \\
\hline Parafovea & $-0.159^{* *}$ & $-0.173^{* * *}$ & $-0.192^{* *}$ & $-0.127^{*}$ \\
\hline Perifovea & $-0.159^{* *}$ & $-0.149^{*}$ & -0.09 & 0.018 \\
\hline \multicolumn{5}{|l|}{ RNFL } \\
\hline Superior quadrant & -0.027 & -0.018 & -0.073 & -0.073 \\
\hline Inferior quadrant & -0.055 & -0.031 & $-0.104^{*}$ & $-0.135^{* *}$ \\
\hline Nasal quadrant & 0.008 & 0.046 & 0.050 & 0.030 \\
\hline Temporal quadrant & -0.022 & -0.041 & -0.085 & -0.028 \\
\hline
\end{tabular}


consequence of the methodologic differences and of the analyzed drugs between these studies, in particular.

Although several risk factors as responsible for the development of HCQ-retinotoxicity, there are controversies concerning the role and dominancy of these factors. ${ }^{5}$ In our study, despite being with lower coefficiency values, we found negative and significant correlations between the thicknesses of retinal layer measured at $1 \mathrm{~mm}$ distant to fovea centralis and drug consumption duration, cumulative dose of drug, dose of drug per kilogram of actual and lean body masses. We excluded the subjects with fundoscopic abnormalities. In addition, HCQ was officially permitted for sale by the government in 2008 in Turkey. Previously, some of the patients bought the drug through importation from other European countries. Therefore, many of the drug-user subjects in our study had a history of drug consumption for less than five years, which was considered as a risk factor for the development of retinotoxicity. As a consequence, we were unable to compare the results of SD-OCT measurements between subjects with and without HCQ-induced retinotoxicity. It is not, thus, reasonable to speculate about factors which may provoke retinotoxicity in the light of our findings.

Although there is a tendency to attribute the higher actual dose, higher cumulative dose and longer duration of drug consumption to the development of retinotoxicity, the fact that shorter duration and lower dose of drug consumption may lead to development of retinotoxicity should not be ignored. ${ }^{21-25}$

On the other hand, there is a controversy about which of the methods is the golden standard for the evaluation of retinotoxicity. ${ }^{26,27}$ In our study, determination of the diminution of the retinal layers by SD-OCT without fundoscopic alterations may lead to drawing attention to evaluation of the role of SD-OCT in further studies. The SD-OCT seems to be an advantageous technique for the evaluation, since it is an easy and fast-applicable technique, gives precise results of entire retinal layers in detail thanks to the high-degree of compatibility among repetitive testing and that it is not affected by the patient-dependent factors. However, it is necessary to standardize and define the cut-off values for the measured thicknesses to define values to be determined in favor of retinotoxicity. Based on the present findings, it is reasonable to evaluate the parafoveal layer by serial SD-OCT.

In our study, patients with connective tissue disease had thinner retinal layers. Whether the reason for these findings was the natural course of the disease or the effect of HCQ treatment was unable to be identified. We believe that the ideal control group for this evaluation should be patients with connective tissue disease who are not under treatment with anti-malarial drugs.

In conclusion, we found that some of the retinal layers were affected by $\mathrm{HCQ}$ even without any signs of retinotoxicity by other modalities. This study is a cross-sectional investigation to obtain general data about retinal involvement by HCQ consumption. The major methodologic flaw is the inability to compare results of retinotoxicity-developed subjects with HCQ users with intact retina. In this concept, we consider the findings of our study as the background data for the next stage of investigation by which we plan to evaluate the effects of drugs on higher cumulative doses and longer duration of consumption in the coming years. Evaluation of a larger group of subjects compared to previous studies is the major superiority of this investigation. Further investigations, which should be performed by longer-duration of drug consuming subjects, preferentially with larger series are necessary to define probable risk factors for the development of retinotoxicity.

\section{Acknowledgements}

We have special thanks to nurse teams of our Rheumatology and Ophthalmology departments due to their valuable contributions during the management of patients evaluation.

\section{Declaration of conflicting interests}

The authors declared no conflicts of interest with respect to the authorship and/or publication of this article.

\section{Funding}

The authors received no financial support for the research and/or authorship of this article. 


\section{REFERENCES}

1. Yam JC, Kwok AK. Ocular toxicity of hydroxychloroquine. Hong Kong Med J 2006 12:294-304.

2. Korah S, Kuriakose T. Optical coherence tomography in a patient with chloroquine-induced maculopathy. Indian J Ophthalmol 2008;56:511-3.

3. Wojtkowski M, Srinivasan V, Fujimoto JG, Ko T, Schuman JS, Kowalczyk A, et al. Three-dimensional retinal imaging with high-speed ultrahigh-resolution optical coherence tomography. Ophthalmology 2005;112:1734-46.

4. Pasadhika S, Fishman GA. Effects of chronic exposure to hydroxychloroquine or chloroquine on inner retinal structures. Eye (Lond) 2010;24:340-6.

5. Marmor MF, Carr RE, Easterbrook M, Farjo AA, Mieler WF; American Academy of Ophthalmology. Recommendations on screening for chloroquine and hydroxychloroquine retinopathy: a report by the American Academy of Ophthalmology. Ophthalmology 2002;109:1377-82.

6. Hangai M, Ojima Y, Gotoh N, Inoue R, Yasuno Y, Makita $\mathrm{S}$, et al. Three-dimensional imaging of macular holes with high-speed optical coherence tomography. Ophthalmology 2007;114:763-73.

7. Ooto S, Hangai M, Sakamoto A, Tomidokoro A, Araie $\mathrm{M}$, Otani T, et al. Three-dimensional profile of macular retinal thickness in normal Japanese eyes. Invest Ophthalmol Vis Sci 2010;51:465-73.

8. Stepien KE, Han DP, Schell J, Godara P, Rha J, Carroll J. Spectral-domain optical coherence tomography and adaptive optics may detect hydroxychloroquine retinal toxicity before symptomatic vision loss. Trans Am Ophthalmol Soc 2009;107:28-33.

9. Weiner A, Sandberg MA, Gaudio AR, Kini MM, Berson EL. Hydroxychloroquine retinopathy. Am J Ophthalmol 1991;112:528-34.

10. Rosenthal AR, Kolb H, Bergsma D, Huxsoll D, Hopkins JL. Chloroquine retinopathy in the rhesus monkey. Invest Ophthalmol Vis Sci 1978;17:1158-75.

11. Mahon GJ, Anderson HR, Gardiner TA, McFarlane S, Archer DB, Stitt AW. Chloroquine causes lysosomal dysfunction in neural retina and RPE: implications for retinopathy. Curr Eye Res 2004;28:277-84.

12. Tehrani R, Ostrowski RA, Hariman R, Jay WM. Ocular toxicity of hydroxychloroquine. Semin Ophthalmol 2008;23:201-9.

13. Rodriguez-Padilla JA, Hedges TR 3rd, Monson B, Srinivasan V, Wojtkowski M, Reichel E, et al. High-speed ultra-high-resolution optical coherence tomography findings in hydroxychloroquine retinopathy. Arch Ophthalmol 2007;125:775-80.
14. Kellner S, Weinitz S, Kellner U. Spectral domain optical coherence tomography detects early stages of chloroquine retinopathy similar to multifocal electroretinography, fundus autofluorescence and near-infrared autofluorescence. $\mathrm{Br} \mathrm{J}$ Ophthalmol 2009;93:1444-7.

15. Wetterholm DH, Winter FC. Histopathology of chloroquine retinal toxicity. Arch Ophtalmol 1964;71:2-7.

16. Bernstein HN, Ginsberg J. The pathology of chloroquine retinopathy. Arch Ophthalmol 1964;71:238-45.

17. Kahn JB, Haberman ID, Reddy S. Spectral-domain optical coherence tomography as a screening technique for chloroquine and hydroxychloroquine retinal toxicity. Ophthalmic Surg Lasers Imaging 2011;42:493-7.

18. Hood DC, Seiple W, Holopigian K, Greenstein V. A comparison of the components of the multifocal and full-field ERGs. Vis Neurosci 1997;14:533-44.

19. Asayama K. [In vivo study on the absorption of the subretianl fluid. 2. Studies on an absorption of tracers (I125.human serum albumin and lanthanum nitrate) injected between the sensory retina and the pigment epithelium layer (author's transl)]. Nihon Ganka Gakkai Zasshi 1976;80:598-607. [Article in Japanese]

20. Bunt-Milam AH, Saari JC, Klock IB, Garwin GG. Zonulae adherentes pore size in the external limiting membrane of the rabbit retina. Invest Ophthalmol Vis Sci 1985;26:1377-80.

21. Bonanomi MT, Dantas NC, Medeiros FA. Retinal nerve fibre layer thickness measurements in patients using chloroquine. Clin Experiment Ophthalmol 2006;34:130-6.

22. Semmer AE, Lee MS, Harrison AR, Olsen TW. Hydroxychloroquine retinopathy screening. $\mathrm{Br} \mathrm{J}$ Ophthalmol 2008;92:1653-5.

23. Warner AE. Early hydroxychloroquine macular toxicity. Arthritis Rheum 2001;44:1959-61.

24. Bienfang D, Coblyn JS, Liang MH, Corzillius M. Hydroxychloroquine retinopathy despite regular ophthalmologic evaluation: a consecutive series. J Rheumatol 2000;27:2703-6.

25. Rüther K, Foerster J, Berndt S, Schroeter J. Chloroquine/ hydroxychloroquine: variability of retinotoxic cumulative doses. Ophthalmologe 2007;104:875-9.

26. Missner S, Kellner U. Comparison of different screening methods for chloroquine/hydroxychloroquine retinopathy: multifocal electroretinography, color vision, perimetry, ophthalmoscopy, and fluorescein angiography. Graefes Arch Clin Exp Ophthalmol 2012;250:319-25.

27. Marmor MF. Comparison of screening procedures in hydroxychloroquine toxicity. Arch Ophthalmol 2012;130:461-9. 